\title{
Supramolecular Electropolymerization
}

\author{
Thomas K. Ellis, ${ }^{\S}$ Melodie Galerne, ${ }^{\S}$ Joseph J. Armao IV, Artem Osypenko, David Martel, Mounir \\ Maaloum, Gad Fuks, Emilie Moulin, Odile Gavat, and Nicolas Giuseppone*
}

\begin{abstract}
Gaining control over supramolecular polymerization mechanisms is of high fundamental interest to understand selfassembly and self-organization processes at nanoscale. It is also expected to significantly impact the design and improve the efficiency of advanced materials and devices. Up to now, supramolecular polymerization has been shown to take place from unimers in solution, mainly by variations of temperature or of concentration. Here we show that supramolecular nucleation-growth of triarylamine monomers can be triggered by electrochemistry in various solvents. The involved mechanism offers new opportunities to precisely address in space and time the nucleation of supramolecular polymers at an electrode. To illustrate the potential of this methodology, we grow and orient supramolecular nanowires over several tens of micrometers in between different types of commercially available electrodes submitted to a single DC electric field, reaching a precision unprecedented in the literature.
\end{abstract}

Because supramolecular bonds are labile in essence, supramolecular polymers are highly dynamic chemical objects. ${ }^{[1,2]}$ The kinetics of formation and dissociation of such polymers can be partially controlled by intensive parameters (e.g. temperature, concentration, mechanical stress) or by modifying in situ their supramolecular recognition units. ${ }^{[3]}$ This toolbox is of particular interest for the design of responsive materials. ${ }^{[4,5]}$ However, one drawback of such an intrinsic dynamics is that gaining control over their polydispersity index is particularly difficult. To improve that aspect, recent seminal works have been developed to access living supramolecular polymerization processes. ${ }^{[6,7]}$ Another very challenging aspect of supramolecular polymerization concerns its precise control in space (i.e. with a spatial resolution approaching the size of the polymer itself). In this direction, the possibility to precisely address the nucleation and growth of supramolecular polymers by electrochemistry attracted our attention.

The rational design of the present study rests on the supramolecular polymerization of triarylamine molecules (TAA) substituted with (an) amide function(s), such as molecule $\mathbf{1}$ in Figure $1^{[8-10]}$ We have previously shown that, in chlorinated solvents, light-triggered oxidation of TAA to the corresponding radical cation $\left(\mathrm{TAA}^{++}\right)$can promote supramolecular polymerization The associated mechanism was thoroughly investigated by a number of experimental and theoretical means, ${ }^{[11,12]}$ and it was proved to involve a nucleation/growth process composed of four elementary steps: ( $I$ ) photoexcitation of a catalytic amount of triarylamines and subsequent oxidation to their radical cations,

[a] Dr. Thomas K. Ellis, Melodie Galerne, Dr. Joseph J. Armao IV, Dr. Artem Osypenko, Dr. David Martel, Prof. Dr. Mounir Maaloum, Dr. Gad Fuks, Dr. Emilie Moulin, Odile Gavat, and Prof. Dr. Nicolas Giuseppone*

SAMS research group - University of Strasbourg - Institut Charles Sadron, CNRS - 23 rue du Loess, BP 84047, 67034 Strasbourg Cedex 2, France

E-mail: giuseppone@unistra.fr

Website: http://sams.ics-cnrs.unistra.fr/

$\S \quad$ These authors contributed equally to this work.

Supporting information for this article is given via a link at the end of the document. with concomitant reduction of the chlorinated solvent producing chloride counterions; (ii) formation of a nucleus of triarylammonium radicals in a double columnar arrangement involving hydrogen bonds; (iii) stacking of neutral triarylamines onto the nucleus and subsequent growth of the primary fibril; (iv) lateral secondary aggregations of fibrils by van der Waals forces to reach larger bundles of fibers.

Based on this mechanism, we envisioned to suppress the lightirradiation step and to replace it by a direct electrochemical oxidation (Figure 1a). We first checked the possibility to oxidize 1 using light irradiation in the presence of a high concentration of commonly used electrolytes $([1]=1 \mathrm{mM}$; [electrolyte] $=100 \mathrm{mM}$ in 1,1,2,2-tetrachloroethane (TCE)). By probing the system with UV-Vis-NIR spectroscopy, we confirmed the effective photoinduced oxidation in the presence of tetrabutylammonium perchlorate $\left(\mathrm{TBAClO}_{4}\right)$, hexafluorophosphate $\left(\mathrm{TBAPF}_{6}\right)$, or chloride (TBACl) (section S5.1, Figure S5). Indeed, as already described extensively, ${ }^{[8,11]}$ one of the typical signatures associated to radical cation $\mathbf{1}^{++}$, and further to the self-assembly process, is the appearance of a stable absorption band around $750 \mathrm{~nm}$ (Figure 1b, dashed line). We then performed cyclic voltammetry (CV) at a scan rate of $100 \mathrm{mV} \cdot \mathrm{s}^{-1}$ for TAA 1 in TCE and in the presence of $\mathrm{TBAPF}_{6}$ (Figure 1c), showing a first oxidation associated with radical cation $1^{1++}\left(E_{1 / 2}=-71 \mathrm{mV}\right)$, and a second oxidation leading to the corresponding dication $\mathbf{1}^{2+}\left(E_{1 / 2}=\right.$ $704 \mathrm{mV}) .{ }^{[13]}$ Spectroelectrochemistry was also performed on this solution, showing the expected UV-Vis spectrum of $1^{\text {*+ }}$ as it passes over the first oxidation potential (Figure 1d). The resulting spectrum perfectly matches with the one observed upon photoirradiation (Figure 1b, plain line). We further tested whether the wires can self-assemble electrochemically from $\mathbf{1}$ and $\mathbf{1}^{++}$in the presence of the electrolyte and in the dark. For that, we probed by ${ }^{1} \mathrm{H}$ NMR spectroscopy the behavior of a solution of $\mathbf{1}$ in deuterated chloroform $(5 \mathrm{mM})$ and in the presence of TBAPF 6 $(100 \mathrm{mM})$, while holding a potential of $0.2 \mathrm{~V} v \mathrm{Fc} / \mathrm{Fc}^{+}$(Ferrocene / Ferrocenium reference redox couple) for $30 \mathrm{~min}$. This time period of electro-oxidation involves the production of approximately $1 \%$ of $\mathbf{1}^{\text {++ }}$ in the solution as determined by coulometry (section S5.2. Figure S7). ${ }^{1} \mathrm{H}$ NMR spectrum shows that, after this electrochemical treatment at constant concentration and temperature, the aromatic proton resonance signals of the triarylamine fully disappear. A similar behavior was observed when electro-oxidation of $\mathbf{1}$ was performed in the absence of electrolyte with a high potential of $25 \mathrm{~V}$ held for $30 \mathrm{~min}$ in TCE- $d_{2}$ (Figure S8a). The disappearance of the NMR signals after the oxidation of a small percentage of the triarylamine molecules is a typical signature of a nucleation step involving radical cations and followed by the formation of large and rigid anisotropic stacks involving all the remaining neutral TAAs as elucidated in our previous works. ${ }^{[8,11]}$

Drop cast samples from this solution were also imaged by both TEM and AFM, confirming the formation of bundled supramolecular triarylamine nanowires (Figure 1e and S8b). By analogy with our previous investigations involving a photogenerated radical cation ${ }^{[11]}$ and accordingly to a series of control experiments (see sections S6-S8 in the supporting information), we can conclude that the electrochemically triggered self-assembly mechanism involves the following sequence 
(Figure 1f): ( $I$ ) diffusion of neutral molecule 1 into the double layer, (ii) oxidation of the neutral species at the electrode surface producing $1^{\mathbf{1}^{+}}$, (iii) migration of the radical cation into the diffusion layer, (iv) nucleation of the radical species, and $(v)$ growth of the supramolecular fibers by addition of neutral monomers onto the nuclei.
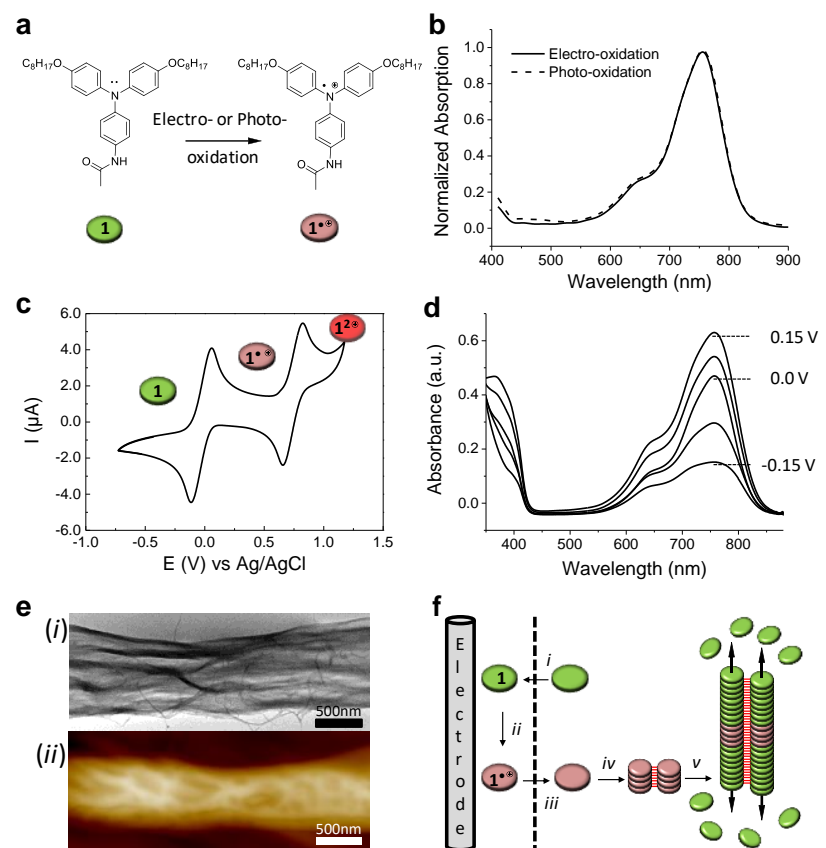

Figure 1. (a) Electro- or photo-triggered oxidation of TAA 1 in its radical cation $1^{\text {*+; }}$ (b) Normalized absorption spectra of electro- and photo-oxidized solutions of 1 at a concentration of $1 \mathrm{mM}$ in TCE and in the presence of $0.1 \mathrm{M} \mathrm{TBAPF}_{6}$; (c) CV data of compound 1 at a concentration of $1 \mathrm{mM}$ in TCE with $100 \mathrm{mM}$ TBAPF $_{6}$ and at a scan rate of $0.1 \mathrm{~V} \cdot \mathrm{s}^{-1}$; (d) Spectroelectrochemical behavior of 1 for different potential scans over the first oxidation wave in the range -0.15 to $0.15 \mathrm{~V}$ vs $\mathrm{Fc} / \mathrm{Fc}^{+}$in TCE with $100 \mathrm{mM} \mathrm{TBAPF}_{6}$; (e) (i) TEM and (ii) AFM height images of the supramolecular fibers formed from $\mathbf{1}$ by an electrochemically triggered process; (f) Simplified electrochemical mechanism of the (nucleation / growth) supramolecular polymerization process activated by electro-oxidation.

Knowing the possibility to electrochemically trigger the nucleation/growth of supramolecular triarylamines in solution, we then investigated the possibility to directly interconnect commercially available microelectrodes in the presence of a sole Direct Current (DC) electric field, without electrolyte. However, when using chloroform or 1,1,2,2-tetrachloroethane to dissolve TAA molecules, we were unable to grow nanowires at low voltages (probably because of the difficulty to form $1^{1^{++}}$in the absence of electrolyte), neither to align them at higher voltages due to short-circuits (probably because of the red-ox activity of the chlorinated solvent itself). Interestingly, we found that TAA 1 can dissolve at elevated temperatures in alkanes (such as heptane in the following study), and that it forms a thermo-responsive fibrillar organogel upon subsequent cooling (with an onset gelation concentration of $2.5 \mathrm{mg} / \mathrm{mL}$ at $\mathrm{T} \approx 10^{\circ} \mathrm{C}$ ) (Figure $2 \mathrm{a}$ ). A peculiar property of this thermal transition is its large hysteresis showing a good stability of the gel when heating up to $\mathrm{T} \approx 30^{\circ} \mathrm{C}$ (Figure S13). By making use of this hysteresis, we found that at $20^{\circ} \mathrm{C}$ the supramolecular fibers formation can be triggered by electroactivation from solution. Indeed, by introducing a $5 \mathrm{mg} / \mathrm{mL}$ solution of 1 between $5 \mu \mathrm{m}$ path sealed ITO plates (which avoids evaporation), and after application of a DC field of $1 \times 10^{7} \mathrm{~V} \cdot \mathrm{m}^{-1}$, we observed the formation of a dense array of fibers by polarized optical microscopy (POM) (Figure 2b). We subsequently questioned whether fibers formation within the electrical field requires an electrical contact at the electrode to start nucleation. A blocked ITO layer device coated with a photoresist did not show fibers formation, except at the edges that contain flaws in the photoresist (Figure S2c). The necessity of the electrical contact for complete wire formation indicates that, even without electrolyte, the immediate contact with the electrode in such high field is able to create an oxidized seeding surface from which neutral TAA can grow. In addition, the continuous growth of individual fibers from the electrode edges across the solution is also highlighting that the fibers formation is clearly not triggered by a sol-gel transition (Figure 2c, and section S10).

We then turned our investigations towards gold interdigitated electrodes (IDEs) of lateral geometry, separated by parallel gaps of $10 \mu \mathrm{m}$, and in the presence of a DC electric field. The substrates were analyzed by POM, SEM, and AFM, all showing the homogeneous alignment of mono-disperse fibers filling all gaps with an orientation parallel to the applied electric field (Figure $2 d-f)$. Using this set-up configuration, we first determined unambiguously that the fibers formation is the result of an electrooxidation and does not come from thermal or concentration effects. Indeed, a first control experiment without an electric field revealed only the presence of amorphous and unlocalized material (Figure S14). Thus, in the time span needed for the solvent to evaporate, the nucleation-growth process does not take place, which also excludes the involvement of dielectrophoretic alignment processes. In a second control experiment, a solution that had been previously allowed to form a gel at low temperature was partly re-solubilized with a gentle heating at $30^{\circ} \mathrm{C}$. At this temperature, although being macroscopically in the sol state, fibers seeds remain in suspension. However, after drop-casting on the IDEs in absence of an electric field and evaporation of the solvent, only a disordered network of fibers was observed (Figure $\mathrm{S} 15 \mathrm{a})$. In addition, application of an electric field of $1.25 \times 10^{7} \mathrm{~V} \cdot \mathrm{m}$ ${ }^{1}$ for duration of 5 minutes after deposition and concentration to the gel state by solvent evaporation, did not show any further alignment (Figure S15b). This last observation indicates that the reorientation of the fibers after entanglement in a low mobility medium is not possible.

Overall, these series of experiments in vertical sealed ITO cells and horizontal gold IDEs configurations reveal that $(i)$ the successful polymerization procedure involves electro-oxidation of TAA 1 at the electrode surface as the sole cause for localized nucleation of fibers, and that (ii) the good alignment of these fibers requires their oriented supramolecular growth in the electric field before the formation of a kinetically trapped disordered entangled network. 


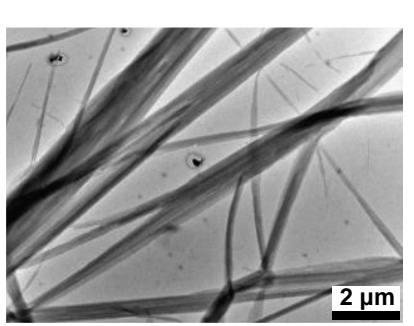

b

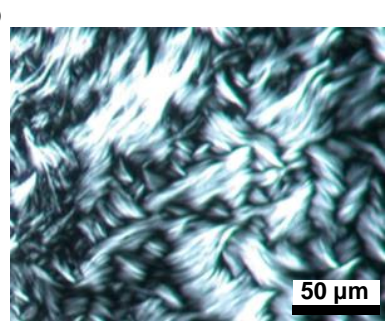

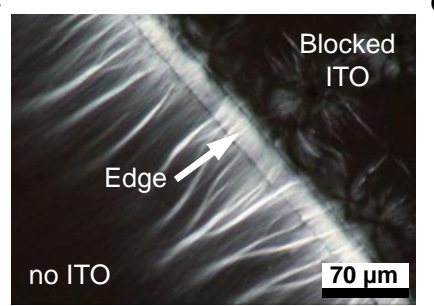

d

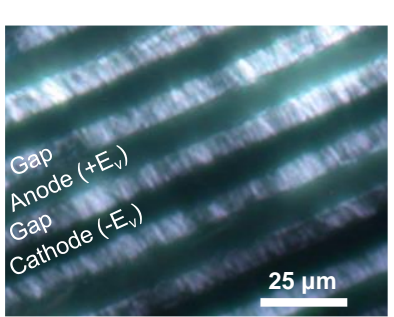

e

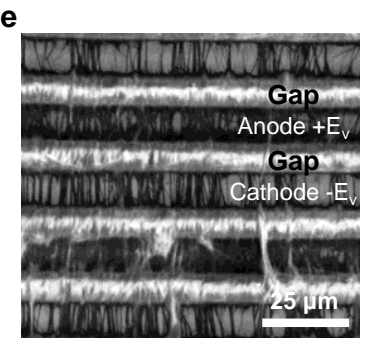

$\mathbf{f}$

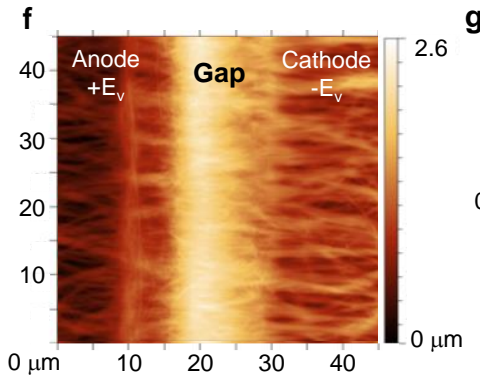

g

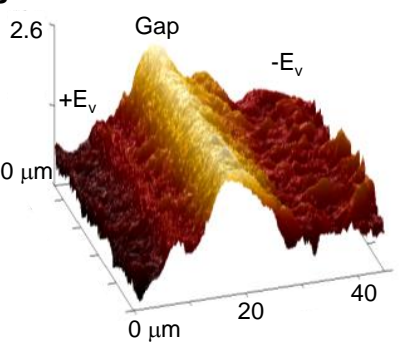

h

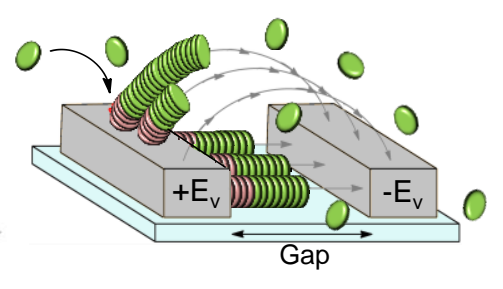

Figure 2. (a) TEM image of the fibrillar organogel of TAA 1 in heptane; (b) Cross-polarized optical microscopy image of supramolecular polymers formed between ITO plates: a $5 \mathrm{mg} / \mathrm{mL}$ solution was let to cool down to room temperature before deposition into the ITO cell with a path of 5 um, and the solution was subsequently subjected to a DC electric field to trigger the supramolecular polymerization; (c) Cross-polarized optical microscopy image of a cell reproducing the protocol described in (b), but using blocked ITO electrodes, avoiding the electrochemically triggered formation of the supramolecular polymers, except at the edge of the ITO domain; (d-f) POM (d), SEM (e) and AFM (f) images of IDEs after drop-casting a $5 \mathrm{mg} / \mathrm{mL}$ solution of 1 and under application of an electric field of $1.25 \times 10^{7} \mathrm{~V} \cdot \mathrm{m}^{-1} ;$ (g) Topological representation of the AFM image shown in (f); (h) 3D schematic depiction of the supramolecular electropolymerization process occurring between IDEs and following the electric field lines as observed in $(\mathrm{g})$ (the green and red ovals represent $\mathbf{1}$ and $\mathbf{1}^{++}$, respectively).

Our description of the self-organization process in the DC field is reinforced by the observation that the nucleation starts always from the anode (+ electrode) with the fibers growing towards the cathode (- electrode), as clearly shown by SEM and AFM on Figures $3 \mathrm{a}$ and $3 \mathrm{c}$. Nucleation/growth at anode was in addition visualized by a real time movie in the interdigitated configuration (Supplementary Movie 1, and section S11.5). Another striking feature of this process is illustrated by the precise topology of the bridging fibers observed by AFM (Figure $2 \mathrm{~g}$ ) and showing that the supramolecular polymers are strictly following the curvature of the electric field during their growth (Figure $2 \mathrm{~h}$ ).

We then studied the effects of field strength and concentration on fibers polymerization and alignment. We determined that the minimum field strength required for the polymerization is $E_{f} \approx 1 \times$ $10^{6} \mathrm{~V} \cdot \mathrm{m}^{-1}$. However, with such a field, the fibers are still highly disordered (Figure S17a,b). Alignment started to occur at $5 \times 10^{6}$ $\mathrm{V} \cdot \mathrm{m}^{-1}$ and was found to be optimal at $1.25 \times 10^{7} \mathrm{~V} \cdot \mathrm{m}^{-1}$ (Figure $\mathrm{S} 17 \mathrm{c}, \mathrm{d})$. For the optimal voltage, we determined that a minimal 2 $\mathrm{mg} / \mathrm{mL}$ concentration of TAA leads to isolated fibers spanning the entire gap (Figure S16). A higher concentration of $5 \mathrm{mg} / \mathrm{mL}$ resulted systematically in fully filled electrode gaps with dense bundles of fibers, with some isolated fibers passing through the cathode (Figure 2e). We quantified the degree of alignment by calculating the order parameter $S(0 \leq \mathrm{S} \leq 1)$ as a function of the electric field value (Figure $3 \mathrm{~b}$, and section $\mathrm{S} 12$ ). At a voltage of $1.25 \times 10^{7} \mathrm{~V} \cdot \mathrm{m}^{-1}$, we found average order parameters of $0.94 \pm 0.08$ for single fibers spanning the entire gap $(2 \mathrm{mg} / \mathrm{mL})$ (Figure $3 \mathrm{~d}$ ) and of $0.87 \pm 0.09$ for single fibers spanning the negative electrodes $(5 \mathrm{mg} / \mathrm{mL}$ ) (Figure S23). This means that high TAA concentration increases the number of fibers spanning the gaps without modifying the very high quality of the alignment. In addition, by applying an electric field to a device with a more complex geometry, that is using circular concentric interdigitated electrodes, we unambiguously proved that the wires precisely follow in any point the field's lines (Figures S18 a-c).

As a last implementation, using microarray electrodes, we were able to grow single supramolecular wires between two pores of less than $5 \mu \mathrm{m}$ in diameter and over a distance of $50 \mu \mathrm{m}$ in length (Figure 3d). The POM image confirms the nucleation from the anode and the fiber growth towards the cathode only when the pores align in the DC field direction. To the best of our knowledge, such a precise bridging configuration is unprecedented in the literature, either by using top-down techniques such as zonecasting $^{[14]}$ or by orienting organogelators ${ }^{[15]}$ and liquid crystal polymers $^{[16-19]}$ in an AC field.

In addition, our previous method consisting in the photo-triggered supramolecular polymerization of triarylamine between nanoelectrodes ${ }^{[20]}$ is not adapted to the various configurations of the present study. First, photo-oxidation appeared experimentally limited to path lengths below $100 \mathrm{~nm}$, a distance almost three orders of magnitude smaller compared to the present devices. We postulate that this limitation partly comes from the necessary use of chlorinated solvents to trigger TAA oxidation with light. Indeed, the large electric field necessary to align the wires on long distances would be shielded by the high amount of photogenerated triarylamonium radical cations and their respective chloride counter ions. If this effect can indeed be overcome by using nanoelectrodes in which the Debye length is larger than the electrode gap, it precludes using photo-oxidation for higher path lengths (section S13). In addition, in our previous approach, ${ }^{[20]}$ fibers were grown everywhere in solution, necessitating the removing of unattached fibers all over the device. Here, making 
use of a single electrical stimulus, the supramolecular electropolymerization mechanism reaches a much higher precision in the sense that fibers can only nucleate from the anode, opening new possibilities to grow selectively single wires between two-point electrodes (as in Figure 3d).

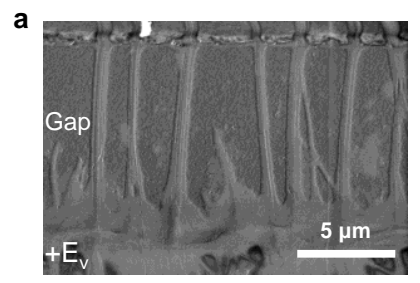

C

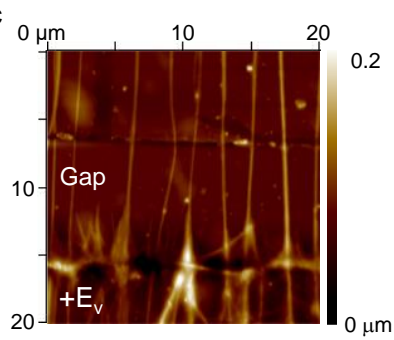

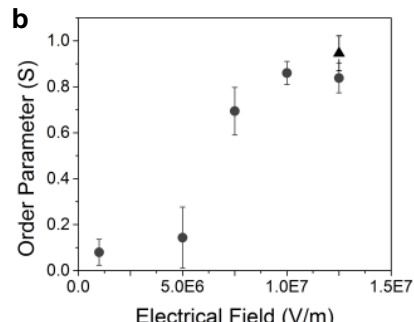

d

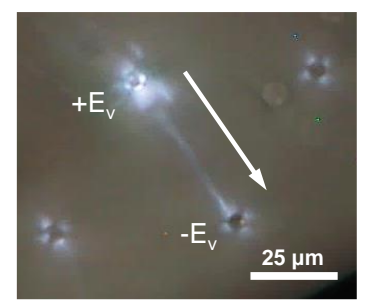

Figure 3. (a) SEM image of an IDE prepared with a $2 \mathrm{mg} / \mathrm{mL}$ solution of 1 in the presence of an applied field of $1.25 \times 10^{7} \mathrm{~V} \cdot \mathrm{m}^{-1}$ and highlighting the nucleation taking place from the anode only; (b) plot of the order parameter (S) as a function of the field strength (dots: [1] = 5 mg/mL; triangle: [1] $=2 \mathrm{mg} / \mathrm{mL}$ ), the error bars are calculated from four different regions of the IDEs; (c) AFM image of an IDE prepared with a $2 \mathrm{mg} / \mathrm{mL}$ solution of 1 in the presence of an applied field of $1.25 \times 10^{7} \mathrm{~V} \cdot \mathrm{m}^{-1}$ and highlighting the formation of thin fibers with nucleation taking place from the anode only; (d) POM image of a microelectrode array showing a single wire grown from the anode to the cathode, parallel to the electric field (white arrow) and over a distance of $50 \mu \mathrm{m}$.

Finally, from a functional point of view, the mobility of charge carriers within the supramolecular nanowires formed by this technique in interdigitated electrodes was derived from the current vs voltage measurement and by using the space charge limiting approximation ${ }^{[21]}$ (which is justified by the low concentration of ions introduced in the system) (section S14). By assuming the number of wires in the electrodes, and by assuming all of them being connected, we can estimate a minimum value as high as $0.56 \mathrm{~cm}^{2} \cdot \mathrm{V}^{-1} \cdot \mathrm{s}^{-1}$, close to the theoretical prediction published in the literature. ${ }^{[22]}$

In conclusion, we have described the first example of what can be defined as a "supramolecular electropolymerization" process. It represents a new tool of fundamental and practical interest for supramolecular chemistry in general. To this end, we have used triarylamines but one can imagine to extend the concept to other molecules with red-ox functionalities and capable of forming supramolecular polymers or self-assemblies involving charge transfer complexes. ${ }^{[23]}$ In addition, we have shown that the mechanism involved can be used to gain control over the precise placement and orientation of supramolecular polymers in between electrodes with a precision that cannot be reached by other current means, up to the bridging of two electrodes of less than $5 \mu \mathrm{m}$ in diameter and over $50 \mu \mathrm{m}$ in length by a single nanowire. Such a precise control in every aspect of supramolecular polymerization, including localization and orientation on nano- and micrometer scales, is at the moment quite unique in the literature. Other potential implementations resulting from these experiments include for instance: $(I)$ spatial control over self-assembly processes in various optoelectronic devices requiring efficient charge transport and ranging from sensors to solar cells, ${ }^{[24,25]}$ (ii) use of waste-free electrochemistry tools to control (living) supramolecular polymerization or to create out-of-equilibrium redox environments for self-organized systems, ${ }^{[26]}$ and (iii) design of $3 \mathrm{D}$ electrical wiring in organogels.

\section{Acknowledgements}

The research leading to these results has received funding from the European Research Council under the European Community's Seventh Framework Program (FP7/2007-2013) / ERC Starting Grant agreement n`257099 (N.G.), the European Union (FP7/2007-2013, ITN Read), the Agence Nationale de la Recherche (STANWs ANR-11-EMMA-0009) and the international center for Frontier Research in Chemistry (icFRC). We also thank the Centre National de la Recherche Scientifique (CNRS), the COST action (CM 1304), the Laboratory of Excellence for Complex System Chemistry (LabEx CSC), the University of Strasbourg (UdS), and the Institut Universitaire de France (IUF). Part of this work was performed within the framework of the IRTG "Soft Matter Science: Concepts for the Design of Functional Materials" (M.G., E. M. and N.G.). We acknowledge the Electron Microscopy Facility of the Institut Charles Sadron. We finally thank André Schroeder for help with optical microscopy.

\section{Conflict of interest}

The authors declare no conflict of interest.

Keywords: Supramolecular polymers $•$ Electrochemistry $•$ Smart materials $\cdot$ Nanowires $\cdot$ Supramolecular electronics

\section{[1] A. Ciferri, Supramolecular Polymers, CRC Press, 2005}

[2] T. F. A. De Greef, M. M. J. Smulders, M. Wolffs, A. P. H. J. Schenning, R. P. Sijbesma, E. W. Meijer, Chem. Rev. 2009, 109, 5687-5754.

[3] X. Yan, F. Wang, B. Zheng, F. Huang, Chem. Soc. Rev. 2012, 41, 6042-6065.

[4] M. A. C. Stuart, W. T. S. Huck, J. Genzer, M. Müller, C. Ober, M. Stamm, G. B. Sukhorukov, I. Szleifer, V. V. Tsukruk, M. Urban, et al., Nat. Mater. 2010, 9, 101-113.

[5] E. Busseron, Y. Ruff, E. Moulin, N. Giuseppone, Nanoscale 2013, 5, 7098-7140.

[6] J. Kang, D. Miyajima, T. Mori, Y. Inoue, Y. Itoh, T. Aida, Science 2015, 347, 646-651

[7] H. Qiu, Z. M. Hudson, M. A. Winnik, I. Manners, Science 2015, 347, 1329-1332.

[8] E. Moulin, F. Niess, M. Maaloum, E. Buhler, I. Nyrkova, N. Giuseppone, Angew. Chem. Int. Ed. 2010, 49, 6974-6978.

[9] J. J. Armao, M. Maaloum, T. Ellis, G. Fuks, M. Rawiso, E. Moulin, N Giuseppone, J. Am. Chem. Soc. 2014, 136, 11382-11388. 
J. J. Armao, P. Rabu, E. Moulin, N. Giuseppone, Nano Lett. 2016, 16, 2800-2805.

[11] I. Nyrkova, E. Moulin, J. J. Armao, M. Maaloum, B. Heinrich, M. Rawiso, F. Niess, J.-J. Cid, N. Jouault, E. Buhler, et al., ACS Nano 2014, 8, 10111-10124.

[12] N. Jouault, E. Moulin, N. Giuseppone, E. Buhler, Phys. Rev. Lett. 2015, 115, 085501.

[13] E. Busseron, J.-J. Cid, A. Wolf, G. Du, E. Moulin, G. Fuks, M. Maaloum, P. Polavarapu, A. Ruff, A.-K. Saur, et al., ACS Nano 2015, 9, 2760-2772.

[14] W. Pisula, A. Menon, M. Stepputat, I. Lieberwirth, U. Kolb, A. Tracz, H. Sirringhaus, T. Pakula, K. Müllen, Adv. Mater. 2005, 17, 684689.

B. W. Messmore, J. F. Hulvat, E. D. Sone, S. I. Stupp, J. Am. Chem. Soc. 2004, 126, 14452-14458.

[16] R. Kuwahara, S. Fujikawa, K. Kuroiwa, N. Kimizuka, J. Am. Chem. Soc. 2012, 134, 1192-1199.
Chem. 2010, 20, 173-179.

[18] M. Yoshio, Y. Shoji, Y. Tochigi, Y. Nishikawa, T. Kato, J. Am. Chem Soc. 2009, 131, 6763-6767.

[19] L. Sardone, V. Palermo, E. Devaux, D. Credgington, M. de Loos, G. Marletta, F. Cacialli, J. van Esch, P. Samorì, Adv. Mater. 2006, 18, 1276-1280.

[20] V. Faramarzi, F. Niess, E. Moulin, M. Maaloum, J.-F. Dayen, J.-B. Beaufrand, S. Zanettini, B. Doudin, N. Giuseppone, Nat. Chem. 2012, 4, 485-490.

[21] A. Rose, Phys. Rev. 1955, 97, 1538-1544.

[22] A. Akande, S. Bhattacharya, T. Cathcart, S. Sanvito, J. Chem. Phys. 2014, 140, 074301.

[23] K. Jalani, S. Dhiman, A. Jain, S. J. George, Chem. Sci. 2017, 8, 6030-6036.

[24] A. Jain, S. J. George, Mater. Today 2015, 18, 206-214.

[25] E. Moulin, J.-J. Cid, N. Giuseppone, Adv. Mater. 2013, 25, 477-487.

[26] R. Merindol, A. Walther, Chem. Soc. Rev. 2017, 46, 5588-5619. 


\section{Entry for the Table of Contents}

\section{COMMUNICATION}

The direct electrochemical oxidation of triarylamines at anodes triggers their nucleation and growth into supramolecular polymers. This mechanism can be defined as a supramolecular electropolymerization process. It is used to grow and precisely address single nanowires over a distance of $50 \mu \mathrm{m}$ between two electrodes in a DC electric field.

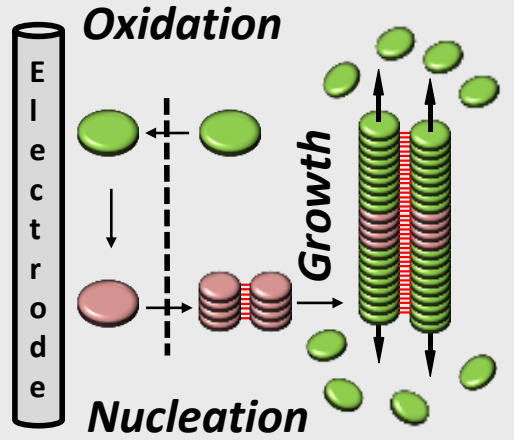

Thomas K. Ellis, Melodie Galerne, Joseph J. Armao IV, Artem Osypenko, David Martel, Mounir Maaloum, Gad Fuks, Emilie Moulin, Odile Gavat, and Nicolas Giuseppone*

Page No. - Page No.

Supramolecular Electropolymerization 